\title{
Reference materials (RMs) for analysis of the human factor II (prothrombin) gene G20210A mutation
}

\author{
Christoph L. Klein ${ }^{1, *}$, János Márki-Zay ${ }^{1}$, \\ Philippe Corbisier ${ }^{1}$, David Gancberg ${ }^{1}$, Susan \\ Cooper $^{2}$, Donato Gemmati ${ }^{3}$, Walter-Michael \\ Halbmayer ${ }^{4}$, Steve Kitchen ${ }^{2}$, Béla Melegh ${ }^{5}$, \\ Michael Neumaier ${ }^{6}$, Johannes Oldenburg ${ }^{7}$, \\ Elisabeth Oppliger Leibundgut ${ }^{8}$, Pieter $\mathrm{H}$. \\ Reitsma ${ }^{9}$, Sandra Rieger ${ }^{10}$, Heinz G.
}

Schimmel ${ }^{1}$, Michael Spannagl ${ }^{11}$, Attila Tordai ${ }^{12}$, Alberto Tosetto ${ }^{13}$, Sophie Visvikis ${ }^{14}$, Renata Zadro $^{15}$ and Christine Mannhalter ${ }^{10, * *}$

${ }^{1}$ Institute for Reference Materials and Measurements, European Commission, Joint Research Centre, Geel, Belgium

${ }^{2}$ Department of Coagulation, Sheffield Haemophilia and Thrombosis Centre, Royal Hallamshire Hospital, Sheffield, United Kingdom

${ }^{3}$ Haemostasis and Thrombosis Study Centre, University of Ferrara, Ferrara, Italy

${ }^{4}$ Central Laboratory, Municipal Hospital Vienna-

Lainz, Vienna, Austria

${ }^{5}$ Department of Medical Genetics and Child Development, University of Pécs, Pécs, Hungary ${ }^{6}$ Institute for Clinical Chemistry, University Hospital Mannheim, University of Heidelberg, Germany ${ }^{7}$ Institute of Transfusion Medicine and Immunohaematology, DRK Blood Donor Service Baden-Wurttemberg/Hessen, Frankfurt am Main, Germany

${ }^{8}$ Laboratory of Molecular Diagnostics, Division of Hematology, University Hospital of Berne, Berne, Switzerland

${ }^{9}$ Laboratory for Experimental Internal Medicine, Academic Medical Centre, University of Amsterdam, Amsterdam, The Netherlands

${ }^{10}$ Clinical Institute of Medical and Chemical Laboratory Diagnostics, Medical University Vienna, Vienna, Austria

${ }^{11}$ Haemostasis Division, University Hospital Munich, Germany

*Corresponding author: Christoph L. Klein, European Commission, Joint Research Centre, Institute for Reference Materials and Measurements, Retieseweg 111, 2440 Geel, Belgium

Phone: +32-14-571634, Fax: +32-14-571548,

E-mail: christoph.klein@cec.eu.int

${ }^{*} \mathrm{CM}$ is the past chairperson of the Committee of Molecular Biology Techniques in Clinical Chemistry (C-MBT) of the International Federation of Clinical Chemistry and Laboratory Medicine (IFCC). CM initiated the project for production of a certified reference material using the prothrombin G20210A mutation as a model system, together with members of C-MBT and on behalf of the IFCC. The Scientific Division of IFCC supported the project ideally and by financial funds. The manuscript has been approved by members of the Scientific Division.
12 Laboratory of Molecular Genetics, National Medical Centre, Institute of Hematology and Immunology, Budapest, Hungary

${ }^{13}$ Hematology Department, S. Bortolo Hospital, Vicenza, Italy

${ }^{14}$ INSERM U525 Faculté de Pharmacie, Nancy,

France

${ }^{15}$ Clinical Institute of Laboratory Diagnosis, University School of Medicine and Clinical Hospital Centre Zagreb, Zagreb, Croatia

\section{Abstract}

The Scientific Committee of Molecular Biology Techniques (C-MBT) in Clinical Chemistry of the IFCC has initiated a joint project in co-operation with the European Commission, Joint Research Centre, Institute of Reference Materials and Measurements to develop and produce plasmid-type reference materials (RMs) for the analysis of the human prothrombin gene G20210A mutation. Although DNA tests have a high impact on clinical decision-making and the number of tests performed in diagnostic laboratories is high, issues of quality and quality assurance exist, and currently only a few RMs for clinical genetic testing are available. A gene fragment chosen was produced that spans all primer annealing sites published to date. Both the wild-type and mutant alleles of this gene fragment were cloned into a pUC18 plasmid and two plasmid RMs were produced. In addition, a mixture of both plasmids was produced to mimic the heterozygous genotype. The present study describes the performance of these reference materials in a commutability study, in which they were tested by nine different methods in 13 expert laboratories. This series of plasmid RMs are, to the best of our knowledge, the first plasmid-type clinical genetic RMs introduced worldwide.

Keywords: metrological traceability; molecular diagnostic testing; mutation; polymerase chain reaction; prothrombin; recombinant DNA; reference standards.

\section{Introduction}

Recent progress in molecular pathology and biotechnology has made DNA-based assays important tools in patient care. DNA tests allow the detection of genetic alterations responsible for inheritable disease conditions, higher risk of developing diseases or altered drug effects. These tests are increasingly used for diagnosis, risk stratification and choice of treatment for patients. DNA test results can be predictive 
for future onset of disorders prior to the manifestation of clinical symptoms, and thus have significant implications for the individual patient and the sibship. Often, testing is only carried out once in a lifetime, and therefore, incorrect genotyping results can have dramatic consequences for the person tested. Thus, quality issues and standardisation of DNA tests are particularly important. Depending on the system used, relatively high error rates for clinical genetic testing have been reported (1-4), specifically including thrombophilic mutations, such as the prothrombin G20210A mutation $(5,6)$. To overcome these issues, the quality of testing has to be assured and improved.

Certified reference materials (CRMs) are recognised as an excellent tool for checking analytical accuracy and are valuable in creating crucial reference points in the development of comprehensive measurement systems (7). CRMs are needed to achieve traceability and comparability of test results and harmonisation of measurement systems. Suitable reference materials (RMs) are widely used as tools for quality assurance (QA). The in vitro Diagnostic Medical Device (IVD) Directive of the EC $(98 / 79 / E C)$ (8) has been in force since December 2003. It requires IVD manufacturers to assure the traceability of values assigned to calibrators and control materials through available reference measurement procedures and/or available reference materials of a higher order. The current lack of CRMs for molecular genetic tests leads to assays not traceable to an accepted common standard. Therefore, the Scientific Committee of Molecular Biology Techniques in Clinical Chemistry (C-MBT) of the International Federation of Clinical Chemistry and Laboratory Medicine (IFCC) in co-operation with the European Commission, Joint Research Centre, Institute for Reference Materials and Measurements (IRMM) initiated a joint project to develop and produce RMs for the analysis of the human prothrombin gene G20210A mutation. In parallel, batches of the described RMs were produced as candidate CRMs, which will be provided as frozen material. These are currently undergoing an IRMM certification process. These clinical genetic RMs are among the first RMs introduced worldwide in this area. Its characteristics and performance in a collaborative field trial are described and discussed in the present article.

\section{Background}

\section{Selection of prothrombin G20210A mutation as target}

The G20210A mutation in the factor II (prothrombin) gene was discovered in 1996 by Poort et al. (9). It is due to a $G \rightarrow$ A transition at base pair 20210 in the $3^{\prime}$ untranslated region of the prothrombin gene. The heterozygous mutation occurs with a frequency of approximately $2-5 \%$ in the Caucasian population. Carriers of this mutation have significantly higher mean plasma prothrombin levels (9). Since the first publication of the G20210A mutation, it has been demonstrated in a number of publications that this genetic variant is a significant and independent risk factor for venous thromboembolism (VTE) (10). The clinical significance of this mutation, the indications for testing and the therapeutic consequences have been elucidated (11).

Analysis of this single nucleotide polymorphism (SNP) is one of the most commonly applied genetic tests in clinical laboratories. Several methods are used for testing, and commercial kits are increasingly applied.

\section{Formats of clinical genetic RMs}

There are many possible formats of genetic RMs, including CRMs: cell lines, genomic DNA, recombinant DNA, synthetic DNA or PCR products, all differing from typical clinical material for investigation. In the present study, RMs for QA were produced as freeze-dried plasmid materials. The advantages of plasmid-type RMs are easy and economical production in large quantities, high reproducibility, versatility, and applicability for qualitative analysis. The main drawback of these genetic RMs is that they cannot be used to assess the quality of DNA extraction procedures. In addition, the length of the fragments inserted is limited and commutability will be impaired if such RMs are applied to methods that use primers outside of the fragment sequence. RMs must have a reasonable shelf life to be useful. Therefore, RM stability is of central importance and has to be guaranteed by the producer (12).

\section{Materials and methods}

\section{Origin of extracted DNA}

The prothrombin gene fragment was selected in the Molecular Biology Division, Department of Clinical Chemistry and Laboratory Medicine, Medical University Vienna, Vienna, Austria. To achieve the highest possible level of test compatibility and commutability, the fragment chosen spans all primer annealing sites published to date (GenBank accession number M17262, nt 26302-26910). Using suitable primers (Pro-fw, 5'-gCACAgACggCTgTTCTCTT-3'; and Pro-rev, 5'-CCCgAgTgCTCggACTACCA-3', synthesised by VBC Genomics, Vienna, Austria, HPLC-purified) a PCR fragment was generated from coded samples of individuals previously tested by other methods and known to carry no mutation or to carry the $G \rightarrow A$ mutation at position 20210 (nt 26784 in the GenBank sequence), respectively.

The individuals had given informed consent to genetic analysis. The use of coded preserved DNA for means of quality assurance conforms to the current Austrian and European regulations.

\section{Cloning of the factor II gene fragment and processing of the stock plasmids}

After initial expansion and characterisation in a pCR2.1 vector (TA Cloning Kit, Invitrogen $\mathrm{GmbH}$, Lofer, Austria), the prothrombin gene fragments were amplified with the following primers: F2cloning-fw, 5'-CgAgCTCggATCCACTAgTAA3'; and F2cloning-rev, 5'-gCCAgTgTgATggATATCTgC-3' 
(Proligo Biochemie GmbH, Hamburg, Germany) and subcloned into the non-patented pUC18 plasmid using the Clontech AdvanTAge PCR Cloning Kit (BD Biosciences Benelux N.V., Erembodegem, Belgium) according to the instructions of the manufacturers.

The resulting new plasmids were called pIRMM-0001 and pIRMM-0002, containing the wild-type or mutant sequence of the factor II gene fragment, respectively. These plasmids were introduced into and amplified in the TOP10 competent E. coli strain provided in the cloning kit.

\section{Amplification and production of stock plasmids}

Streaks of bacterial colonies were tested by PCR for the presence of the inserts. The orientation of the inserts was assessed by digestion with Hindlll. A single clone containing the wild-type or mutant fragment, respectively, in the same orientation was selected and used as the source of the plasmid stocks for the CRMs.

Plasmid stocks were obtained after amplification overnight in liquid LB broth medium (LB Ampicillin-100 Broth; Fluka, Sigma, Bornem, Belgium) containing $100 \mu \mathrm{g} / \mathrm{mL}$ ampicillin and purification using a Qiagen Plasmid Maxi Kit (Qiagen, Westburg, Leusden, Netherlands).

\section{DNA quantitation}

The stock solution (Tris-HCl) of plasmid DNA was quantified using the PicoGreen ${ }^{\circledR}$ dsDNA quantitation kit (Molecular Probes, VWR International, Leuven, Belgium) according to the manufacturer's instructions. The copy number of the plasmids in the stock solution was calculated on the basis of DNA concentration.

\section{Sequencing of the stock plasmids}

The factor II gene inserts in the stock plasmids were sequenced for confirmation using the primers (Pro-fw and Pro-rev) described above and CEO Dye Terminator Cycle Sequencing Kit with the Beckman CEO2000XL Sequencer (Beckman Coulter Inc.) according to the manufacturer's instructions.

\section{Production of pIRMM-0001, -0002 and mixed RMs}

Sterile, self-standing, high-recovery $1.5-\mathrm{mL}$ polypropylene vials were filled with either $5 \times 10^{4}$ copies of plasmid pIRMM$0001,5 \times 10^{4}$ copies of pIRMM-0002 or a mixture of $2.5 \times 10^{4}$ copies of pIRMM-0001 and $2.5 \times 10^{4}$ copies of pIRMM-0002 in a volume of $100 \mu \mathrm{L}$ containing $100 \mu \mathrm{g} / \mathrm{mL}$ bovine serum albumin (BSA) as additive. These samples were freeze-dried (FD) in a CHRIST LPC-16 EPS 2-65DS freeze-dryer, and the vials were stored at $-20^{\circ} \mathrm{C}$.

The Quality Management System of the IRMM Reference Materials Unit is in compliance with the requirements of ISO Guide 34 and ISO 17025. Characterisation of RMs is performed according to the requirements given by ISO Guide 35.

\section{Commutability study}

Commutability as a property of genetic RMs can be described as showing behaviour similar to usual clinical samples giving the true result when investigated by different routine genotyping procedures. To evaluate the commutability of the RMs, a field study was performed. In the absence of any reference method for genetic testing, RMs have to be evaluated by a consensus of recognised reference laboratories.
Table 1 Concentrations measured by the PicoGreen method and copy numbers of the stocks of plasmids pIRMM-0001 (wild-type) and pIRMM-0002 (containing the G20210A mutation).

\begin{tabular}{lll}
\hline Plasmid & $\begin{array}{l}\text { DNA } \\
\text { concentration, } \\
\mu \mathrm{g} / \mathrm{mL}\end{array}$ & $\begin{array}{l}\text { Copy number, } \\
\mathrm{cp} / \mathrm{mL}\end{array}$ \\
\hline pIRMM-0001 & 306 & $8.48 \times 10^{13}$ \\
plRMM-0002 & 298 & $8.25 \times 10^{13}$ \\
\hline
\end{tabular}

Copy numbers were calculated from the concentration values on the basis of the size of the plasmids (3310 bp for both).

A total of 16 leading laboratories in the field of testing for genetic mutations in coagulation factor genes were invited to participate in the field study. Of these expert laboratories, 13 responded positively and participated in the study.

Two vials of each genotype and two vials without DNA, but containing the RM matrix, giving a total of eight vials, were coded, graded randomly, named as Samples A-H, and sent on ice to each laboratory for testing.

The testing laboratories were provided with the study protocol, the terms to be used for reporting, and the result reporting form, but had no information on the expected results. In the study protocol, the aim of the study, properties of samples (content and minimal sample intake, stability and storage, warning to users), preparation (reconstitution) of the materials and reporting requirements were described in detail. The following details were required on the result reporting form: title and address of the laboratory; number of genetic tests performed yearly (factor II G20210A and in general); involvement in proficiency testing programmes for Factor II G20210A mutation and the relevant organisation; dates of arrival and testing; storage conditions; and incidental observations on the samples. The laboratories were asked to describe the methods used in detail and to report the genotyping results in a standardised form. Further comments on results were welcomed.

\section{Results}

Analysis of the different colonies revealed that all clones contained the plasmids. DNA concentrations of the plasmid preparations were evaluated by PicoGreen quantitation. The results and calculated copy numbers are listed in Table 1.

\section{Sequencing}

Sequence analysis of the selected fragments showed that the isolated 609-bp sequences were identical to the GenBank reference sequence (accession number M17262, nt 26302-26910), except for the G20210A mutation (corresponding to nt 26784 in the reference sequence) and an $A \rightarrow G$ point mutation at position 20054 (nt 26628 in the GenBank reference sequence). Interestingly, this second mutation was already present in the patient sample and was linked to the mutated sequence. The cloned wild-type fragment corresponded to $100 \%$ of the reported prothrombin sequence. A map of the pUC18 plasmids produced is shown in Figure 1. 


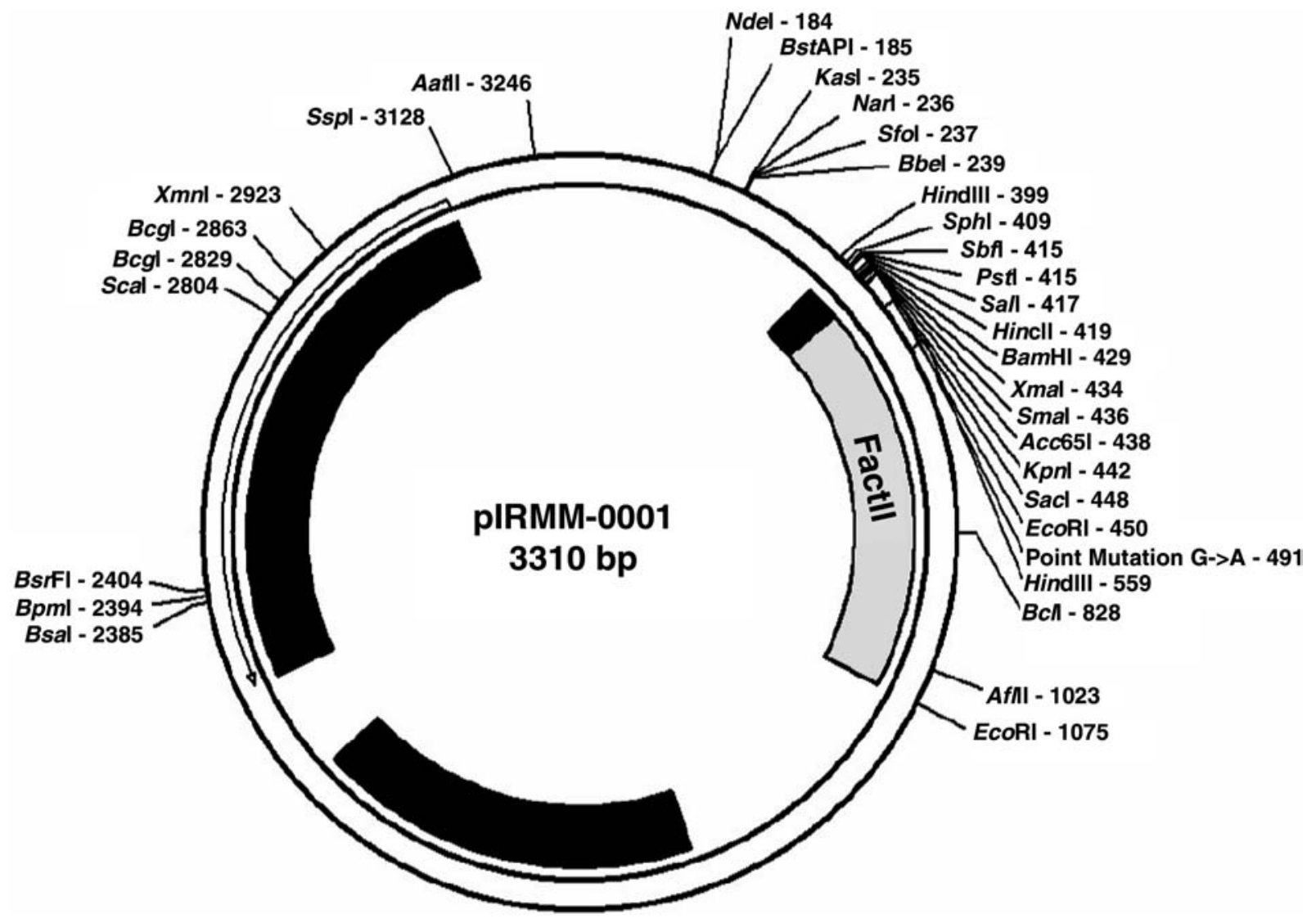

Figure 1 Map of plasmid pIRMM-0001. pIRMM-0001 and pIRMM-0002 are pUC18 plasmids containing the EcoRI fragment of the factor II gene insert from plasmid pCR2.1. The factor II mutation G20210A is located at position 491. Plasmid pIRMM-0001 contains $\mathrm{G}$ at the position corresponding to the wild-type sequence; pIRMM-0002 reads $A$ at the same locus, representing the G20210A mutation.

\section{Commutability study}

For analysis of the samples, nine different methods were used; two of the laboratories applied two different methods, which were reported separately. The method used most often was real-time PCR using the LightCycler (LC) system and kit commercially available from Roche (Roche Diagnostics Belgium, Vilvoorde, Belgium). This method is based on the fluorescence resonance energy transfer (FRET) tech-

Table 2 Methods used in the testing laboratories for determination.

\begin{tabular}{|c|c|c|c|}
\hline Laboratory code & Method(s) & Reference(s) and remark(s) & Primers \\
\hline 1 & LightCycler, Roche kit & Confidential, not communicated & \\
\hline 2 & LightCycler, Roche kit & 32 cycles instead of the original 45 & \\
\hline 3 & PCR-RFLP (HindIII) & $\begin{array}{l}\text { Poort SR et al. (9), modified using an } \\
\text { elongated mutagenic primer }\end{array}$ & A-fw, A-rev \\
\hline 4 & $\begin{array}{l}\text { LightCycler, Roche kit; } \\
\text { PCR-RFLP (HindIII) }\end{array}$ & $\begin{array}{l}\text { Confidential, not communicated } \\
\text { Poort et al. (9) }\end{array}$ & B-fw, B-rev \\
\hline 5 & PCR-RFLP (HindIII) & Danneberg et al. (13) & C-fw, C-rev \\
\hline 6 & PCR-RFLP (HindIII) & In-house method, not published earlier & D-fw, D-rev \\
\hline 7 & $\begin{array}{l}\text { LightCycler, see reference; } \\
\text { PCR-RFLP (HindIII) }\end{array}$ & $\begin{array}{l}\text { Aslanidis et al. (14) } \\
\text { Hybridisation probes: E-det and E-anch } \\
\text { Poort et al. (9) }\end{array}$ & $\begin{array}{l}\text { E-fw, E-rev; } \\
\text { B-fw, B-rev }\end{array}$ \\
\hline 8 & LightCycler, Roche kit & Confidential, not communicated & \\
\hline 9 & Taq-Cycle-Sequencing & $\begin{array}{l}\text { ABI PRISM } 310 \text { Genetic Analyser and } \\
\text { Big Dye Terminator Kit (Applied } \\
\text { Biosystems, Darmstadt, Germany) }\end{array}$ & F-fw, F-rev \\
\hline 10 & PCR-RFLP (HindIII) & Poort et al. (9) & B-fw, B-rev \\
\hline 11 & Mutagenically separated PCR & Endler et al. (15) & $\begin{array}{l}\text { G-fw, G-revA } \\
\text { G-revG }\end{array}$ \\
\hline 12 & LightCycler, Roche kit & Confidential, not communicated & \\
\hline 13 & Mutagenically separated PCR & $\begin{array}{l}\text { In-house method developed with } \\
\text { an industrial partner, confidential }\end{array}$ & \\
\hline
\end{tabular}

Primer and probe sequences known are described in Figure 2. 


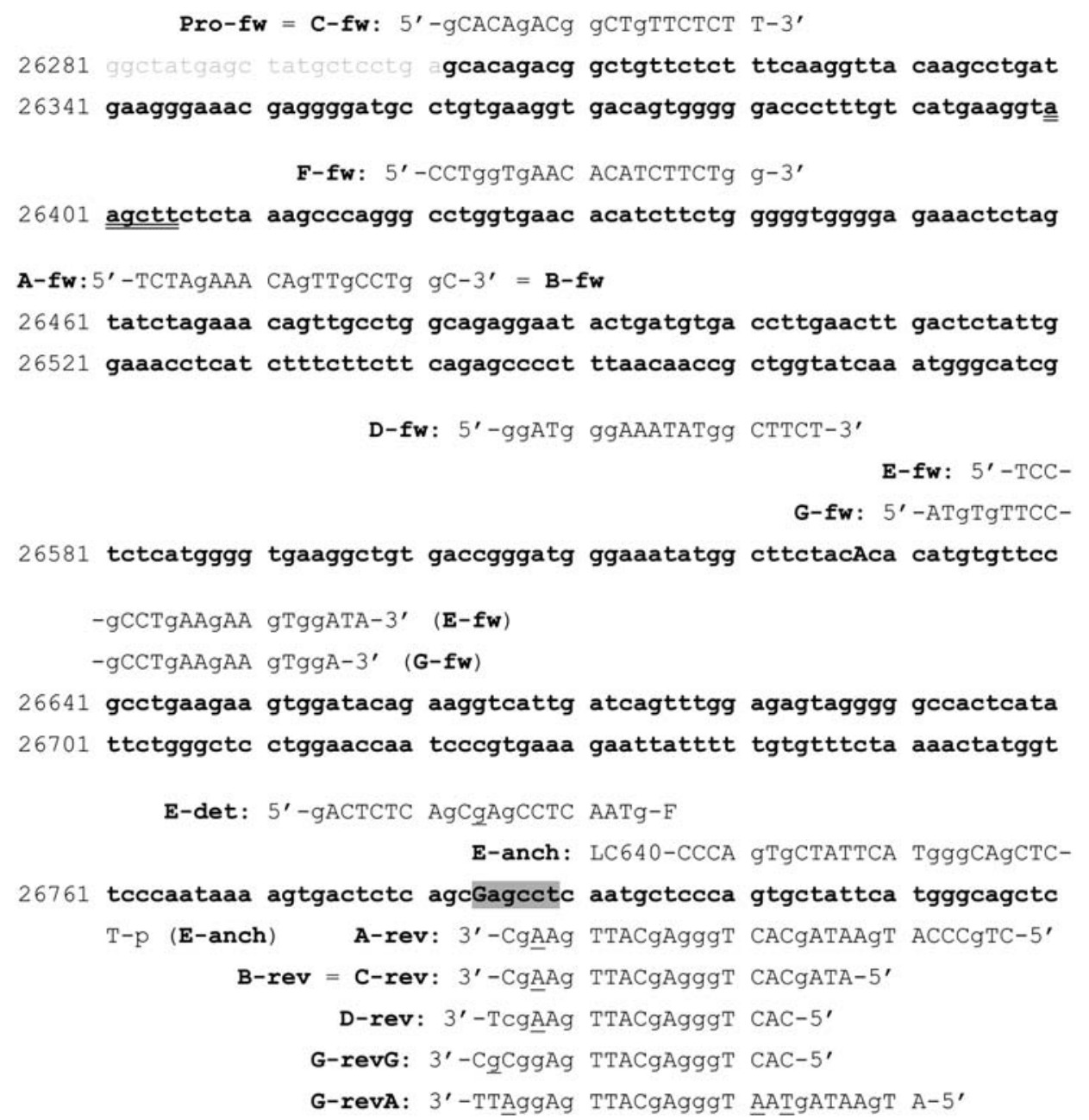

26821 tctgggctca ggaagagcca gtaatactac tggataaaga agacttaaga atccaccacc

F-rev: $3^{\prime}$-ATTCT TAggTggTgg-

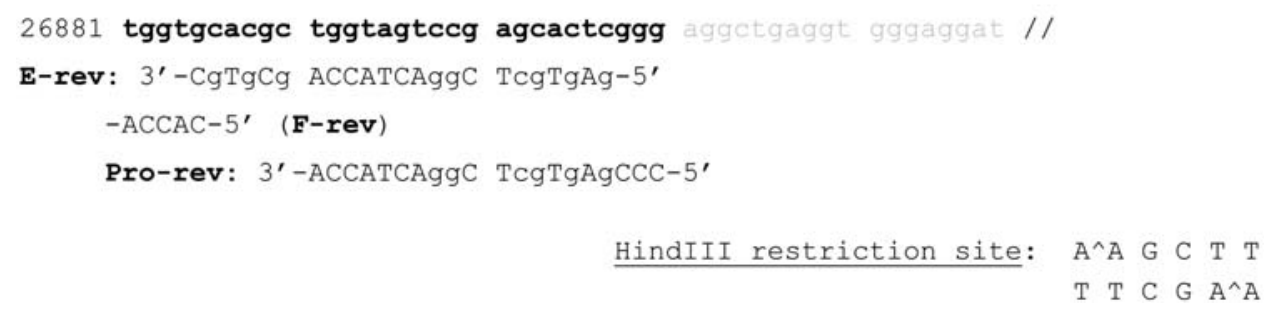

HindII restriction site: $A^{\wedge} A$ G C T T

Figure 2 Reference sequence of the prothrombin gene fragment chosen (nt 26302-26910, GenBank accession number M17262) with the positions of primers and probes used by different testing laboratories. The sequence of the wild-type gene fragment inserted fully corresponds to the reference sequence represented by bold characters with position numbers at the beginning of the lines. The G20210A mutation (position nt 26784) is indicated by a capital G. The A $\rightarrow \mathrm{G}$ variation at $\mathrm{nt} 26628$ found in the mutated fragment has not been described previously. The position of this mutation is marked by a capital A. It is linked to the G20210A mutation in the selected DNA. Primers and probes used by the testing laboratories are positioned at their annealing sites and marked with the abbreviations used in Table 2. Mismatching nucleotides of PCR primers are underlined. PCR-RFLP methods published use Hindlll digestion for mutation detection following the introduction of a restriction site in the presence of mutation by mutagenic primers. This potential restriction site is highlighted in grey. The prothrombin gene contains another Hindlll restriction site close to the G20210A mutation, which can be used as an internal control for Hindlll digestion (13). This site is double-underlined.

nology. One laboratory used the LightCycler with an in-house FRET method. Six laboratories used PCR amplification followed by Hindlll digestion, and two laboratories used multiplex PCR with allele-specific primers.

Every RM was genotyped correctly and no amplification or genotype was reported for the blank sam- ples. However, one laboratory that used the Roche commercial kit for the LightCycler with a reduced number of cycles (32 instead of 45 recommended in the kit) reported a low signal intensity. When the test was repeated using 45 cycles, the intensity was appropriate. For the mixed RM, to mimic the heterozygous genotype, it was reported that signal intensi- 
ties for both alleles were similar for the different methods.

The testing methods are listed in Table 2. The primers and probes with known sequences used by the testing laboratories are listed in Figure 2.

\section{Discussion}

Suitable CRMs are urgently needed in the field of human genetic testing $(16,17)$.

The advantages of plasmid-type RMs include easy and economical production in large quantities, high reproducibility, versatility, and applicability for qualitative analysis. The major drawback of these genetic RMs is that they cannot be used to assess the quality of DNA extraction procedures. In addition, the length of the fragments inserted is limited. Cloned plasmidtype RMs contain only one defined specific sequence, and similar sequences, which might be of importance when using a certain method, would not be within the scope of the investigation. This restricted commutability represents one limit for the wider use of such RMs.

Assays for defined point mutations, such as the G20210A mutation in the prothrombin gene, usually amplify short fragments, and thus the problem can be tackled by appropriate selection of the fragment inserted into the plasmid.

Most genetic tests for the detection of mutations or polymorphisms are qualitative rather than quantitative - their purpose is to determine the presence or absence of a particular DNA sequence. Accordingly, the concentration of the target sequence is not a key issue, but quantification was the basis for checking for appropriate copy numbers in the RMs. Considering the uncertainty of this calculation and possible DNA degradation during the freeze-drying and reconstitution process, the concentration $\left(5 \times 10^{4}\right.$ copies/vial) given is only a nominal value, supplied as additional material information.

It was demonstrated in the study that the concentration of such plasmid DNA is not critical, as the detection limit of the testing methods is high and low concentrations of test material can usually be compensated by an increase in cycle numbers. Even under assay conditions optimised for high DNA concentrations of test material, when the RMs did not give satisfactory signal intensity, the sample volume could be increased at the expense of water in the PCR test to correct for weak signals. Consequently, in future production of CRMs, the copy numbers could be increased to allow sample sizes of $1 \mu \mathrm{L}$.

From another point of view, such RMs could be a possible source of contamination in PCR analysis if not handled appropriately. Obviously, RMs presented as PCR products or plasmids are good templates for most of the amplification primers utilised and may require specific handling procedures. Appropriate preservation of the RM to keep the copy number of the target sequence low represents an efficient tool to reduce contamination risk.
Sequence analysis confirmed that the reference sequences of the RMs produced are identical to the GenBank reference sequence, except for the G20210A mutation and an $A \rightarrow G$ point mutation at position 20054 in the mutated sequence. This second mutation was linked to the mutated sequence and was already present in the patient sample, which means that it was not introduced during production. To the best of our knowledge, this sequence change has not been reported previously and any associations with pathological conditions are unknown. Recently, an $A \rightarrow G$ exchange at position 19911 has been identified. This mutation seems to affect the splicing efficiency of prothrombin (18). The A20054G mutation did not cause any problems during production of the plasmid-type RMs or in the field study. However, difficulties may arise when a primer is used that binds with its $3^{\prime}$ end to this sequence. Therefore, this sequence alteration is reported in the documentation of the CRMs.

Comparable studies are being performed within the framework of a shared cost project, which is partly funded by the European Commission under FP5 "CRMGEN", in which IRMM is a partner. This is a feasibility study for the development of different RM prototypes in the field of genetic testing, such as plasmid-type RMs.

\section{Conclusion}

In conclusion, in the present study initiated by the IFCC C-MBT, it could be shown that plasmid-type RMs containing defined point mutations can be produced and successfully used in tests for detection of point mutations in a typical QA scheme. At present, the corresponding CRMs have been produced as frozen material, which will be delivered under defined conditions at $-20^{\circ} \mathrm{C}$ until a successful long-term stability study has been carried out to determine the success of freeze-drying techniques. Whether or not the procedure is also suitable for RMs (including CRMs) containing repetitive sequences of variable length still needs to be evaluated in depth.

\section{Acknowledgements}

The project was supported by a grant from the IFCC and complementary finance from the European Commission JRC Institute for Reference Materials and Measurements. A. Torday was supported by Hungarian Scientific Research Fund (OTKA) T034830 and by National Research and Development Programmes (NKFP) 1/024/2001.

\section{References}

1. Dequeker E, Ramsden S, Grody WW, Stenzel TT, Barton DE. Quality control in molecular genetic testing. Nat Rev Genet 2001;2:717-23. 
2. Dequeker E, Cassiman JJ. Evaluation of CFTR gene mutation testing methods in 136 diagnostic laboratories: report of a large European external quality assessment. Eur J Hum Genet 1998;6:165-75.

3. Dequeker E, Cassiman JJ. Genetic testing and quality control in diagnostic laboratories. Nat Genet 2000; 25:259-60.

4. Losekoot M, Bakker B, Laccone F, Stenhouse S, Elles R. A European pilot quality assessment scheme for molecular diagnosis of Huntington's disease. Eur J Hum Genet 1999;7:217-22.

5. Tripodi A, Peyvandi F, Chantarangkul V, Menegatti M, Mannucci PM. Relatively poor performance of clinical laboratories for DNA analyses in the detection of two thrombophilic mutations - a cause for concern. Thromb Haemost 2002;88:690-1.

6. Neumaier M, Braun A, Gessner R, Funke H. Experiences with external quality assessment (EQA) in molecular diagnostics in clinical laboratories in Germany. Working Group of the German Societies for Clinical Chemistry (DGKC) and Laboratory Medicine. Clin Chem Lab Med 2000;38:161-3.

7. Müller MM. Implementation of Reference Systems in Laboratory Medicine. Clin Chem 2000;46:1907-9.

8. EU Lex. Directive 98/79/EC of the European Parliament and of the Council on in vitro Diagnostic Medical Devices of 27 October 1998. Off J Eur Comm 1998;L331:1-37.

9. Poort SR, Rosendaal FR, Reitsma PH, Bertina RM. A common genetic variation in the 39-untranslated region of the prothrombin gene is associated with elevated plasma prothrombin levels and an increase in venous thrombosis. Blood 1996:88:3698-703.

10. Endler G, Mannhalter C. Polymorphisms in coagulation factor genes and their impact on arterial and venous thrombosis. Clin Chim Acta 2003;330:31-55.
11. McGlennen RC, Key NS. Clinical and Laboratory Management of the Prothrombin G20210A Mutation. Arch Pathol Lab Med 2002;126:1319-25.

12. Pauwels J, Schimmel H, Lamberty A. Criteria for the certification of internationally acceptable reference materials. Clin Biochem 1998;31:437-9.

13. Danneberg J, Abbes AP, Bruggeman BJ, Engel H, Gerrits $\mathrm{J}$, Martens A. Reliable genotyping of the G-20210-A mutation of coagulation factor II (prothrombin). Clin Chem 1998;44:349-51.

14. Aslanidis C, Nauck M, Schmitz G. High-speed prothrombin $\mathrm{G} \rightarrow \mathrm{A} 20210$ and methylenetetrahydrofolate reductase $\mathrm{C} \rightarrow \mathrm{T} 677$ mutation detection using real-time fluorescence PCR and melting curves. Biotechniques 1999;27:234-8.

15. Endler G, Kyrle PA, Eichinger S, Exner M, Mannhalter C. Multiplexed mutagenically separated PCR: simultaneous single-tube detection of the factor $V$ R506Q (G1691A), the prothrombin G20210A, and the methylenetetrahydrofolate reductase A223V (C677T) variants. Clin Chem 2001; 47:333-5

16. Ibarreta D, Elles R, Cassiman JJ, Rodriguez-Cerezo E, Dequeker E. Towards quality assurance and harmonization of genetic testing services in the European Union. Nat Biotechnol 2004;10:1230-5.

17. Barton D, Klein CL, Stacey G. Certified reference materials for genetic testing. In: Fuchs J, Podda M, editors. Encyclopedia of diagnostic genomics and proteomics. New York: Marcel Dekker, 2005:226-31.

18. von Ahsen N, Oellerich $\mathrm{M}$. The intronic prothrombin $19911 \mathrm{~A} \rightarrow \mathrm{G}$ polymorphism influences splicing efficiency and modulates effects of the $20210 \mathrm{G} \rightarrow$ A polymorphism on mRNA amount and expression in a stable reporter gene assay system. Blood 2004;103:586-93.

Received March 7, 2005, accepted June 17, 2005 